\title{
Andreu Castell
}

\section{Deutsch und Deutschunterricht aus spanischer und katalanischer Sicht}

\begin{abstract}
Dass Spanien nicht gerade als Beispiel für die konsequente und andauernde Förderung des Fremdsprachenlernens gelten kann, dürfte allgemein bekannt sein. Genauso wie die Tatsache, dass sich weder die Zentralregierung noch die Landesregierungen in mehr als 20 Jahren für eine klare und rationale Fremdsprachenpolitik haben entscheiden können. Und trotzdem haben die Leute in Spanien Fremdsprachen gelernt, unter anderem auch Deutsch, einige haben sogar Germanistik studiert. Wenn man vor einigen Jahren noch in der Lage war oder zumindest versuchte, sich Hoffnungen zu machen, steht man gegenwärtig vor einer nicht zu übersehenden und zum Teil auch noch nicht völlig erklärten Tatsache. Ein Rückgang der Schüler- bzw. Studentenzahlen macht sich zwar bezüglich aller Fremdsprachen bemerkbar, er betrifft aber die deutsche Sprache vielleicht noch stärker. Gründe dafür kann es natürlich viele und verschiedene geben. Hängt es mit den sich überall bemerkbar machenden Folgen des Geburtenrückgangs zusammen? Spielt es eine Rolle, dass Teile Spaniens über eine eigene Landessprache verfügen? Hängt es damit zusammen, dass das Deutsche bisher ja auch noch weniger gefördert worden war als beispielsweise das Englische oder das Französische? Was hat die spanische Germanistik dagegen getan? Was die deutsche Regierung? Wie stehen die Verlage dazu? All dies sind Fragen, die seit langem offen stehen, die uns aber gerade jetzt in noch größerem Maße beschäftigen sollten. Fragte man sich noch vor Kurzem, ob wir nicht auf dem Wege der Besserung seien, sehen wir uns jetzt vor die Frage gestellt, ob es überhaupt noch einen Ausweg gibt.
\end{abstract}

„IT IS everywhere. Some $380 \mathrm{~m}$ people speak it as their first language and perhaps twothirds as many again as their second. A billion are learning ist, about a third of the world's population are in some sense exposed to it and by 2050 , it is predicted, half the world will be more or less pfoficient in it. It ist the language of the globalisation - of international bussiness, politics and diplomacy. It is the language of computers and the Internet. You'll see it on posters in Côte d'Ivoire, you'll hear it in pop songs in Tokyo, you'll read it in official documents in Phnom Penh. Deutsche Welle broadcast in it. Bjork, an Icelander, sings in it. French business schools teach in it [...]" (The Economist, 20.12.2001).

Es wäre ein Zeichen kindlicher Unschuld meinerseits, Sie jetzt raten zu lassen, auf welche Sprache sich der Autor der vorangehenden Zeilen bezieht. Denn noch ist es ja nicht das Spanische.

In der spanischen Tageszeitung La Vanguardia vom 11. Januar 2002 verglich der katalanische Journalist Carlos Sentís mit unversteckter Ironie den spanischen Wirtschaftsminister Rodrigo Rato, den Wirtschaftskommissar 
der EU Pedro Solbes - ebenfalls Spanier - und den Vorsitzenden der Europäischen Zentralbank Wim Duisenberg mit drei Musketieren, die sich während ihrer Pressekonferenz am 4. Januar problemlos und korrekt in gerade derselben Sprache äußerten, um die positiven Daten bezüglich der ersten Schritte des neugeborenen Euros bekannt zu geben. Niemand habe, wunderte sich der Journalist, etwas dagegen eingewendet, obwohl es eigentlich irgendwie komisch habe wirken müssen, gerade dabei exklusiv in dieser Sprache sprechen zu hören, wo das Vereinigte Königreich sich doch freiwillig aus der Eurozone ausgeschlossen habe.

Nun, wie sowohl die Mitarbeiter von The Economist, als auch Carlos Sentís und wir selber alle wissen, beruht der Erfolg der englischen Sprache ja nicht so sehr auf den Verdiensten unserer nahen Vettern, sondern vielmehr auf der politischen, wirtschaftlichen und technischen Macht des großen Bruders jenseits des Atlantiks. Nach dem Eurobarometer 55 (Oktober 2001) ${ }^{1}$ haben nur $16 \%$ der EU-Bürger das Englische als Muttersprache. Zählt man jedoch Muttersprache und erste Fremdsprache zusammen, dann wird Englisch von $48 \%$ der EU-Bürger benutzt. Es folgt Deutsch mit $32 \%$, Französisch mit $26 \%$, Italienisch mit $18 \%$ und Spanisch mit $15 \%$, um nur die Sprachen zu erwähnen, die von mehr als $10 \%$ der EU-Bürger verwendet werden.

Deutsch ist also, hinter Englisch, die in der EU am meisten gesprochene Sprache. Vergleicht man es mit Französisch, fällt aber sofort auf, dass dieser zweite Platz durch die Zahl der eigentlichen Muttersprachler bedingt ist, die im Falle von Deutsch $24 \%$ ausmachen, bei Französisch lediglich $15 \%$. Dafür benutzen $8 \%$ der EU-Bürger das Deutsche und $11 \%$ das Französische als Fremdsprache. Woraus man folgern könnte, dass das Interesse für Französisch als Fremdsprache unter den EU-Bürgern größer ist als das für das Deutsche.

Verfolgt man das Eurobarometer von Oktober 2001 weiter, bemerkt man natürlich, dass eine solche Folgerung zu pauschal ist, da das Interesse für eine bestimmte Fremdsprache stark vom jeweiligen Land abhängt. So trifft sie allem Anschein nach nur für die Mittelmeerländer zu, mit Ausnahme von Griechenland, wo Deutsch beliebter zu sein scheint als Französisch. Was Spanien anbelangt, das sich übrigens nach dem Vereinigten Königreich als das fremdsprachenfaulste EU-Land entpuppt, ${ }^{2}$ erweist sich unsere Folgerung nach den Angaben im Eurobarometer als richtig. Spanier halten Französisch für nützlicher als Deutsch. Man könnte meinen, das habe auch seine Richtigkeit und seinen guten Grund. Eine langjährige Tradition (bis zu den 70er Jahren war Französisch die erste Fremdsprache im spanischen Schulsystem,

I Siehe <http://europa.eu.int/comm/dg10/epo/eb/eb55/eb55.html> (eingesehen am 8.3.2002).

${ }^{2}$ Man könnte sich fragen, in welchem Maße die 40 Jahre Abgeschiedenheit während Francos Diktatur einen Einfluss darauf ausgeübt haben und welche Rolle derzeitig die Mehrsprachigkeit Spaniens selbst und das vor allem in letzter Zeit immer stärker werdende Bewusstsein, Spanisch sei ja selbst eine Weltsprache, dabei spielen. 
weit vor Englisch), Spanien grenzt an Frankreich, und zudem ist Französisch eine romanische und daher auch zumindest im Prinzip eine leichter zu erlernende Fremdsprache als z. B. Englisch oder gar Deutsch.

Im Juli 1998 gab die damalige spanische Erziehungsministerin Esperanza Aguirre die Absicht der Regierung bekannt, Englisch schon ab dem 1. Schuljahr in der Primarstufe anzubieten und dazu auch noch eine zweite Fremdsprache als Pflichtfach in der Sekundarstufe einzuführen. Sie fügte, natürlich zur Empörung aller Germanistenverbände, hinzu, dabei werde es sich im Allgemeinen um Französisch handeln, obwohl es natürlich auch Deutsch oder Italienisch sein könnte.

In Einklang damit: Im Jahr zuvor waren in Andalusien für die Sekundarstufe neben 40 Lehrstellen für Englisch, 130 für Französisch und keine einzige für Deutsch ausgeschrieben worden. In Galicien wurden in den Jahren 1999 und 2000 jeweils 60 und 70 Lehrstellen für Französisch ausgeschrieben, ebenfalls keine einzige für Deutsch. Und das in zwei autonomen Regionen, die über Kulturhoheit und außerdem über zwei wichtige Germanistik-Institute verfügen, die jährlich eine ganze Reihe von möglichen Deutschlehrern für die Sekundarstufe ausbilden. In Katalonien, wo ebenfalls an der Universität Barcelona Germanisten ausgebildet werden, war es etwas anders. 1997 wurden für die Sekundarstufe 10 Lehrstellen für Französisch und 2 für Deutsch ausgeschrieben, in den Jahren 1998 und 1999 waren es jeweils jedes Mal 15 für Französisch und 2 für Deutsch. Der Unterschied ist zwar nicht mehr so gravierend wie in Andalusien und Galicien, aber immerhin stehen wir im Falle der autonomen Region, in der vielleicht am meisten Deutsch unterrichtet wird, weiterhin vor einem Verhältnis von fast 8:1 zugunsten des Französischen. Entspricht das der tatsächlichen Nachfrage?

Wohl kaum, wie es aus verschiedenen Tatsachen und Informationsquellen zu entnehmen ist. Ich habe rund 1.300 Stellenangebote in der Sonntagsausgabe der spanischen Tageszeitung El País in der Zeit vom 1. Dezember 2001 bis zum 17. Februar 2002 daraufhin ausgewertet, welche Fremdsprachen darin gefordert wurden. Zunächst lässt sich festhalten, dass in $72 \%$ der Fälle explizit Fremdsprachenkenntnisse gefordert wurden. Von diesen wurde in $71,6 \%$ der Fälle lediglich Englisch gefordert; in 3,2 \% nur Deutsch und in $1 \%$ nur Französisch. Zählt man diese Fälle mit denen zusammen, in denen neben Englischkenntnissen auch Deutsch- oder Französischkenntnisse gefordert (4,9\% gegenüber 3,2\%) oder zumindest positiv bewertet wurden $(3,2 \%$ gegenüber 6,5\%), dann ergibt sich für das Französische ein Anteil von 10,7\%, für das Deutsche ein Anteil von 11,3\%. ${ }^{3}$

Schon in El País vom 28. März 1998 hieß es, die Nachfrage nach Französisch als Fremdsprache liege bei 10,6\%, die nach Deutsch bei 9,8\%. Das

${ }^{3}$ Das entspricht bei weitem nicht mehr den Ergebnissen in Ammon (1993, S. 43), wo für Französisch ein Wert von $20,99 \%$ und für Deutsch hingegen ein Wert von lediglich $6,17 \%$ ermittelt wird. 
entspricht größtenteils - wie auch die Ergebnisse bezüglich der Stellenangebote - dem, was man den Zahlen in den sogenannten Offiziellen Sprachenschulen entnehmen kann. Das sind öffentliche Schulen, die der Fortbildung von Jugendlichen und Erwachsenen im Bereich der Fremdsprachen dienen sollen und die - im Rahmen der Möglichkeiten und der bestehenden Nachfrage - insgesamt bis zu fünf aufeinander folgende Jahreskurse für die jeweilige Sprache anbieten. Gerade diese Schulen, die ein hochqualifiziertes, methodologisch meistens modernes und zugleich ein preiswertes Angebot darstellen, können dazu dienen, das tatsächliche Interesse der Spanier für zumindest bestimmte Fremdsprachen zu messen. Im Schuljahr 1999/2000 haben an den katalanischen Offiziellen Sprachenschulen von 24.227 eingeschriebenen Schülern 19,25 \% Französisch und 17,15\% Deutsch gelernt. Betrachtet man lediglich die Zahlen der im ersten Kurs eingeschriebenen, dann entscheiden sich $24 \%$ für Französisch und 23,15\% für Deutsch. ${ }^{4}$ In einer mittelgroßen Schule wie die von Lleida kann man sogar von einer Ausgangsvorliebe für das Deutsche sprechen. Im Durchschnitt für die Schuljahre 1997/1998 bis 2001/2002 entscheiden sich dort die Schüler im ersten Kurs im Allgemeinen eher für das Deutsche als für das Französische $(52,9 \%$ gegenüber $47,1 \%$ ). Bemerkenswert ist allerdings, dass sich das Verhältnis von Kurs zu Kurs umkehrt. Im zweiten Kurs lernen im Durchschnitt für die erwähnten Jahre $40 \%$ Deutsch und $60 \%$ Französisch, im fünften und letzten Kurs machen lediglich $36,4 \%$ mit Deutsch weiter gegenüber $63,6 \%$, die sich bei Französisch noch im Rennen halten. ${ }^{5}$ Deutsch, eine schwierige Sprache?

Wie sieht es nun in der Sekundarstufe aus? Katastrophal. Das Angebot an Deutsch als Fremdsprache an den spanischen Sekundarschulen variiert zwar von einer autonomen Region zur anderen, ist aber überall noch als extrem defizitär zu betrachten; selbst in Regionen wie den Kanarischen Inseln oder Katalonien, wo man von einer relativ großen Zahl von Schulen sprechen kann, die Deutsch anbieten. In Katalonien wird Deutsch als erste oder zweite Fremdsprache durchgängig bis zum Abitur lediglich an 2,5\% der bestehenden Schulen angeboten; als zweite Fremdsprache als Wahlfach an etwa $20 \%$. Man mag denken, Letzteres sei ja im Grunde viel. Das täuscht aber, denn es handelt sich in diesen Schulen, die sowohl öffentlich als auch privat sein können, meistens um ein unregelmäßiges Angebot, das plötzlich und ohne ersichtliche Gründe einfach ausfallen kann, wobei auch noch zu beachten ist, dass der Deutschunterricht an diesen Schulen meist nicht von ausgebildeten Germanisten, - die ja in Spanien theoretisch dazu da sind -, sondern hauptsächlich von Religions-, Latein-, Altgriechisch-, Philosophie- und

${ }^{4}$ Quelle: Departament d'Ensenyament. Servei d'Estadística i Documentació. Estadística de l'Ensenyament. Curs 1999-2000. Escoles Oficials d'Idiomes. Alumnes segons idioma i curs. Total.

${ }^{5}$ Quelle: direkt von der Schulleitung übermittelte Zahlen. 
in einigen Fällen selbst von Französischlehrern erteilt wird, wenn kein Bedarf an ihren eigenen Fächern besteht. Es handelt sich oft um Lehrer, die selbst nur einige Deutschkurse gemacht haben oder die sich beim GoetheInstitut Barcelona haben umschulen lassen und die natürlich auch noch andere Fächer unterrichten. Anfangs standen die katalanischen Universitäten und der Katalanische Germanistenverband diesem Phänomen, das vom Goethe-Institut durch Umschulungsangebote gefördert wurde, sehr skeptisch gegenüber - schließlich verloren dadurch Germanisten Arbeitsmöglichkeiten -, angesichts der Evolution der Dinge sieht man heutzutage darin zumindest die Möglichkeit, somit eine Nachfrage nach Deutsch überhaupt beweisen zu können.

Das Argument, es bestehe im Sekundarbereich an sich einfach keine Nachfrage nach Deutsch, wurde lange Zeit von allen Verwaltungen angeführt. Sowohl das Erziehungsministerium in Madrid als auch die entsprechenden Stellen in den Regionalregierungen, sei es nun in Andalusien, in Galicien, im Baskenland oder in Katalonien, haben dieses Argument nicht selten benutzt. Daneben hieß es dann, sobald es gelang, an der Gültigkeit des Arguments zu rütteln, es gebe einfach kein Geld für mehr Deutschlehrer. Und das ist natürlich ein Argument, gegen das man schwer ankommt. Das zur Zeit noch geltende Schulgesetz zwingt zwar dazu, an den Sekundarschulen eine zweite Fremdsprache anzubieten, es hat aber nicht einen, sondern gleich zwei Haken.

Den ersten stellt die Tatsache dar, dass die 1998 von der damaligen Ministerin ausgesprochene Absicht, eine zweite Fremdsprache als Pflichtfach in der Sekundarstufe einzuführen, noch nicht realisiert worden ist. Dem Schüler steht es weiterhin frei, sich unter den vielen und durchaus verschiedenartigen Wahlfächern, die ihm zur Verfügung gestellt werden, für eine zweite Fremdsprache oder aber vielleicht für einen Tanz- oder Makrameekurs zu entscheiden.

Kommen wir nun zum zweiten und vielleicht noch wichtigeren Haken. Das Gesetz verpflichtet lediglich dazu, eine zweite Fremdsprache anzubieten, es verpflichtet die Schulen aber keineswegs dazu, mehr als eine zweite Fremdsprache anzubieten. Das bedeutet im Endeffekt, dass in den meisten Sekundarschulen einfach nur Französisch als solche angeboten wird, ganz unabhängig davon, ob wirklich Nachfrage besteht oder nicht. Der Grund dafür, den die Verwaltung nicht selten bei Unterredungen mit Verantwortlichen von Germanistenverbänden anführt, ist einfach: Es gebe viele Französischlehrer, die nun schon mal da seien und die eine Investition bedeuteten, die ausgenutzt werden müsse. Wenn man will, ein eigentlich - zumindest aus finanzieller Sicht - ganz plausibler Grund. Es bleibt jedoch eine Frage offen: Wenn es schon so viele Französischlehrer gibt, warum werden dann weiterhin Planstellen für Französisch ausgeschrieben, und dazu auch noch, wie schon erwähnt, so viele wie z. B. in Andalusien oder in Galicien?

Hier scheint das trotz aller Hindernisse nicht nachlassende und durchaus legitime Interesse Frankreichs, das Status des Französischen als Weltsprache 
zu bewahren, eine Rolle zu spielen. Ob sich lediglich Politiker oder auch Unternehmer bei den spanischen Behörden dafür einsetzen, ist mir unbekannt, dass aber jemand es tut, dessen können wir uns gewiss sein. Ganz anders läuft die Sache auf deutscher Seite. Da hat es offiziell immer geheißen, man sehe es ein, aber man wolle sich ja nicht in landesinterne Angelegenheiten einmischen. Zur Förderung des Deutschen sei ja schon das Goethe-Institut da. Und das Goethe-Institut hat gewiss viel getan für das Deutsche in Spanien, aber politischen oder wirtschaftlichen Druck kann es nicht ausüben. Und außerdem gehört auch dort Bescheidenheit und Zurückhaltung zum guten Stil. Angesichts der langsam, aber anscheinend unaufhörlich sinkenden Schülerzahlen wurde vor ein paar Jahren in den beiden Goethe-Instituten in Spanien über neue Werbeformen nachgedacht. Seitens einiger Deutschlehrer kam der Vorschlag, das Deutsche als etwas Besonderes anzubieten und darzustellen, ja sogar als nicht leicht und somit eben als eine Art persönlicher Herausforderung. Damit sollten Schüler selbst, aber vor allem auch Eltern angesprochen werden, denn man hatte beobachtet, dass es in vielen, hauptsächlich privaten Schulen der Primarstufe quasi zu einer Prestigesache geworden war, Deutsch als Fremdsprache anzubieten. ${ }^{6}$ Anscheinend hatte es zu einem Zeitpunkt, wo man buchstäblich um Schüler werben muss, seine Wichtigkeit. Was geschah? Der Vorschlag wurde rundweg abgelehnt, mit dem Argument, es klinge doch zu elitär. Jahrelang hatte das Goethe-Institut mit einer Art Inszenierung für Deutsch geworben, wo in der jeweiligen Sekundarschule selbst eine erste Stunde Deutsch mit einem Zauberer durchgeführt wurde, durch die gezeigt werden sollte, wie lustig und auch wie einfach es doch sei, Deutsch zu lernen. Ganz erfolglos blieb es nicht. Aber könnte der vorhin erwähnte Neuerungsvorschlag - je nach persönlicher ideologischer Gesinnung - vielleicht als politisch unkorrekt eingestuft werden, dann könnte man auch meinen, die bis dahin auf diese Weise durchgeführte Werbung sei vielleicht auch nicht so ganz unschuldig. Dass Werbung es mit der Wahrheit nicht immer ernst nimmt, ist allgemein bekannt. Aber warum soll man den Leuten weismachen, dass Deutsch eine leichte Sprache ist? Warum soll man den Leuten überhaupt weismachen, dass es leicht ist, eine Fremdsprache zu lernen? Seit wann muss denn Lernen leicht sein? Anscheinend in unserer Zeit, wo um alles geworben werden muss.

Und selbst Politiker werben ja andauernd. Im vergangenen Monat wiederholte die jetzige Erziehungsministerin Pilar del Castillo, was schon ihre Vorgängerin vor vier Jahren angekündigt hatte: den Willen der Regierung, die erste Fremdsprache schon im ersten Schuljahr der Primarstufe als Pflichtfach einzuführen - also für Kinder im Alter von sechs Jahren anstatt wie bis jetzt für Kinder im Alter von acht Jahren. Zugleich solle im ersten Schuljahr

${ }^{6}$ Hier darf man keineswegs nur an rein private Schulen denken. Es handelt sich meistens um Schulen, die zwar nicht staatlich sind, die aber vom Staat oder von der jeweiligen autonomen Regierung finanziell unterstützt werden. 
der Sekundarstufe - also für Kinder im Alter von zwölf Jahren - eine zweite Fremdsprache als Pflichtfach eingeführt werden. Man stütze sich dabei, so die Ministerin, auf verschiedene, jedoch nicht explizit erwähnte Studien von Experten, aus denen hervorgehe, dass man eine Fremdsprache am besten zwischen dem dritten und dem zwölften Lebensjahr erlernen könne (El País, 16.2.2002).

Unabhängig davon, ob es diesmal nun wirklich realisiert werden wird oder nicht, halte ich diesen Vorschlag in einem in Bezug auf Fremdsprachenpolitik quasi unterentwickelten Land wie Spanien als reine Propaganda den Eltern gegenüber. Andere Experten vertreten nämlich die Meinung, Fremdsprachenunterricht vor dem neunten Lebensjahr sei in einer Situation, wo der Lerner nur in der Unterrichtsstunde selbst dieser Sprache ausgesetzt sei, größtenteils vergeudete Zeit. ${ }^{7}$ Wir dürfen nicht vergessen, dass die Umstände in Spanien nicht die gleichen sind wie in Ländern, wo z. B. kaum Filme synchronisiert werden und selbst Fernsehsendungen im Original laufen. Es stellt sich somit die Frage, ob es nicht günstiger und vor allem ehrlicher wäre, schon etwas früher mit der zweiten Fremdsprache zu beginnen oder wirklich bilingualen Unterricht zu betreiben, anstatt jetzt noch mehr für Englisch als einfaches Pflichtfach in der Primarstufe zu investieren. ${ }^{8}$ Das Geld, das man dadurch einsparen würde, könnte vielleicht dazu benutzt werden, Deutsch in der Primarstufe anzubieten.

Wie es dort um das Deutsche steht, mag die Tatsache beweisen, dass es selbst in Katalonien im Schuljahr 1999-2000 in keiner einzigen öffentlichen Schule angeboten wurde, weder als erste noch als zweite Fremdsprache. ${ }^{9} \mathrm{Als}$ maßgebend mag auch folgende Tatsache betrachtet werden: 36 Universitäten bieten in Spanien den Studiengang Fremdsprachenlehrer für die Primarstufe an. An zweien werden lediglich Englischlehrer ausgebildet, an allen anderen sowohl Englisch- als auch Französischlehrer. Nur an einer - an der Universität Salamanca in ihrer Niederlassung in Zamora besteht seit zwei Jahren die Möglichkeit, sich als Deutschlehrer ausbilden zu lassen. Wenig versprechend in einem Kontext, in dem sich die europäischen Unterrichtsminister doch zu der sogenannten Muttersprache-plus-zwei-Regel verpflichtet haben. ${ }^{10}$

Keine Schüler - keine Lehrer, keine Lehrer - keine Schüler, ein Teufelskreis, der sich vielleicht bald auch in der Sekundarstufe bemerkbar machen wird. Dort sind es, von den schon erwähnten und durchaus nicht seltenen Ausnahmen abgesehen, Germanisten, die für den Deutschunterricht zustän-

7 Siehe z. B. Muñoz (1999 u. 2001).

${ }^{8}$ Hinsichtlich der neuen Möglichkeiten, die sich in letzter Zeit bezüglich des Deutschen im bilingualen Unterricht in Andalusien ergeben haben, siehe Hengst (2001).

9 Wie schon erwähnt, wird es an Privatschulen doch angeboten.

${ }^{10}$ Ganz unabhängig davon, wie so etwas in schon mehrsprachigen Ländern bzw. Regionen wie Katalonien, Valencia, Galicien und das Baskenland im Falle Spaniens zu interpretieren ist, wo schon eine bestimmte Zahl von Unterrichtsstunden dem Spanischen selbst als erste Fremdsprache gewidmet werden. 
dig sind oder zumindest zuständig sein sollten. Die Zahl der Sekundarschulen, an denen Deutsch als Fremdsprache angeboten wird, hat mit der Zeit, wenig zugenommen, die der Offiziellen Sprachenschulen, in denen auch hauptsächlich Germanisten unterrichten, in relativ starkem Maße - 1990 gab es deren 70, heute sind es schon 172, von denen 121 (70\%) auch Deutsch anbieten. Universitäten, an denen man Deutsch als Fremdsprache lernen kann gibt es viele, " solche, wo Germanistik angeboten wird, lediglich acht: Barcelona, Complutense de Madrid, País Vasco, Salamanca, Santiago de Compostela, Sevilla, Valencia und Valladolid. ${ }^{12}$

Und genau wie selbst in den so beliebten Offiziellen Sprachenschulen, wo man mittlerweile auch einen Rückgang der Schülerzahlen zu melden hat, sind die Studentenzahlen auch an der Universität, und somit auch in der Philologie allgemein, selbst in der englischen, und natürlich auch in der Germanistik gesunken. Dies mag verschiedene Gründe haben. Einerseits die sich schon seit zwei Jahren heftig bemerkbar machenden Folgen des Geburtenrückgangs der vergangenen zwei Jahrzehnte, der nach einigen Berechnungen dazu führen wird, dass sich die Zahl der jetzigen Stundeten - rund 1.500.000 - in acht Jahren um ein Drittel verringern wird. Hinzu kommt, dass sich die Zahl der Studiengänge seit 1987 mehr als verdreifacht hat. ${ }^{13}$ Das bedeutet im Endeffekt, dass immer weniger Studenten sich auf mehr Studiengänge verteilen werden müssen, dass es mittlerweile schon viele Studiengänge gibt, wo es mehr Dozenten als Studenten gibt.

Natürlich sind nicht alle Universitäten und alle Studiengänge in gleichem Maße von diesem Phänomen betroffen, die der Geisteswissenschaften sind es aber auf jeden Fall: In Katalonien z. B. sind es im Studienjahr 2001/02 hauptsächlich Katalanische Philologie (mit einem Rückgang von $38 \% \mathrm{im}$ Vergleich zum vorangehenden Studienjahr), Geschichte (-28,5\%), Geographie $(-26,5 \%)$ und Spanische Philologie $(-19 \%)$. Aber auch nicht geisteswissenschaftliche Fächer wie Physik (-28,5\%) und Mathematik (-17\%) erfahren einen starken Rückgang (La Vanguardia, 9.12.2001). Französische Philologie musste im vergangenen Jahr an der Universität Girona als Vollstudium abgeschafft werden, da sich nur zwei neue Studenten voreingeschrieben hatten. Was Germanistik betrifft, so kann man provisorisch fest-

${ }^{11}$ Im Vorwort zu Acosta (1997) wundert sich J.M. Rodríguez über die große Ausbreitung des Deutschunterrichts an den spanischen Universitäten. Nicht weniger als 15.000 Studenten lernten an ihnen zu dem Zeitpunkt Deutsch. Es handelt sich tatsächlich um eine hohe Anzahl, man darf jedoch nicht außer Acht lassen, was aus der Erhebung von Acosta sehr deutlich hervorgeht, dass rund 13.700 dieser Studenten ein eher kurzfristiges Lernen des Deutschen als Fremdsprache betreiben (1-3 Jahreskurse).

12 An der Universität Rovira i Virgili in Tarragona wird als Aufbaustudium der universitätseigene - also nicht vom zentralen Erziehungsministerium zertifizierte - Studiengang Estudios Alemanes (Deutschlandstudien) angeboten.

13 Es gibt in diesem Augenblick 160. Siehe dazu <http://www.elpais.es/temas/dossieres/ leyuniversidades/cifras 1.html $>$ (eingesehen am 7.3.2002). 
halten, dass sich der Rückgang auch dort bemerkbar gemacht hat. Im Falle der Universität Barcelona könnte man vom Studienjahr 1999/2000 auf das Studienjahr 2000/2001 von einem Rückgang von rund $10 \%$ sprechen (von insgesamt 331 eingeschriebenen Studenten auf insgesamt 299), während z. B. die Universität Valladolid im gleichen Zeitraum einen Rückgang von rund $30 \%$ erfährt (von insgesamt 107 Studenten auf 75). ${ }^{14}$

Man kann es nur als besorgniserregend empfinden, umso mehr als sich diese Entwicklung gerade zu einem Zeitpunkt bemerkbar macht, in dem die spanische Germanistik nicht einfach dahinschlummert. ${ }^{15}$ Noch nie war sie so aktiv gewesen wie in den neunziger Jahren. Das Ungleichgewicht zugunsten der hauptsächlich literarischen Ausrichtung, das es lange Zeit auch in Spanien gegeben hatte, ist seit langem überwunden. ${ }^{16}$ Es stimmt zwar - wie Zurdo (2001, S. 375) mit Recht behauptet -, dass die Erforschung der strukturellen und funktionellen Übereinstimmungen und Verschiedenheiten, welche zwischen der deutschen und der spanischen Sprache festzustellen seien, trotz einiger mehr oder weniger gelungenen Gesamtdarstellungen, eigentlich kaum über Ansätze hinausgekommen seien; aber diese Ansätze haben sich in der erwähnten Zeitspanne herausgebildet, und - was ich eigentlich für noch wichtiger halte - der Vergleich zwischen den beiden Sprachen hat mittlerweile auch schon in den Studienplänen einen mehr oder weniger stark verankerten Platz gefunden, genauso wie Übersetzungskurse und die Didaktik des Deutschen als Fremdsprache. ${ }^{17}$ Für Letzteres waren und sind auch heute noch hauptsächlich die sogenannten Erziehungswissenschaftlichen Institute der Pädagogen zuständig, die - zumindest was DaF betrifft - lange Zeit einfach nur weltfremd danebengearbeitet haben. Nur im Rahmen der Germanistik und in enger Zusammenarbeit mit Lehrkräften aus Sekundarschulen und Offiziellen Sprachenschulen wird es möglich sein, gute Lehrer auszubilden, wenn sich die Verwaltung flexibel genug zeigt, diese Zusam-

${ }^{14}$ Berechnet anhand der Statistiken, die beide Universitäten auf ihrer Webseite anbieten (<http://www.ub.es > und <http://www.uva.es $>$ ) (eingesehen am 5.3.2002).

15 Vgl. dazu Pfeiffer (1998, S. 40).

${ }^{16}$ Das wird zum einen aus den verschiedenen Studienplänen ersichtlich, wo bei den Pflichtfächern im Durchschnitt ein Verhältmis von 1,41:1 zugunsten der linguistisch ausgerichteten Fächer besteht. Umgekehrt sieht es nur an der Universität Sevilla aus, wo sich noch ein Verhältnis von 1,12:1 zugunsten der literarisch und kulturhistorisch ausgerichteten Fächer feststellen lässt. Es folgen mit ansteigendem Verhältnis zugunsten der linguistischen Ausrichtung Barcelona (1,22:1), Complutense de Madrid $(1,32: 1)$, Salamanca $(1,42: 1)$, Valencia $(1,60: 1)$, Valladolid $(1,66: 1)$, Santiago $(1,68: 1)$ und País Vasco $(1,91: 1)$. Zum anderen wird es auch aus der Tatsache ersichtlich, dass von den zwischen 1980 und 2000 verfassten Dissertationen $60 \%$ eher sprachwissenschaftliche Ziele verfolgen. Beschränkt man die Erhebung auf die 90er Jahre, dann lässt sich ein Verhältnis von 2:1 zugunsten der sprachwissenschaftlichen Dissertationen feststellen.

${ }^{17}$ Kontrastive Grammatik wird als Wahlfach an fünf der acht Universitäten angeboten (Complutense de Madrid, Salamanca, Santiago, Sevilla und Valladolid), Übersetzungskurse an allen außer an der Complutense de Madrid und Sevilla - in Valladolid sogar als Pflichtfach - und Didaktik an allen außer in Sevilla und Valladolid. 
menarbeit zu erleichtern und zu fördern, wie es zum Teil z. B. schon in Barcelona, Madrid und País Vasco geschieht, wo von den Universitäten Fortbildungsprogramme auf der Grundlage der regionalen Adaptation der Fernstudienbriefe für das Fach $\mathrm{DaF}$ des Deutschen Instituts für Fernstudien der Universität Tübingen, der Gesamthochschule Kassel und des Goethe-Instituts angeboten werden. ${ }^{18}$

Ernüchternd und - je nachdem, wie man darüber denken mag - pessimistisch klingt im Zusammenhang mit der Motivation, im Ausland Deutsch zu lernen oder gar noch Germanistik zu studieren, die Formulierung von Stassen (2001, S. 6 u. 11) bezüglich der Situation in den Jahren 1997/1998:

„Standen früher die ästhetische (Deutsch als Kultursprache) und die epistemologische (Deutsch als Wissenschaftssprache) Motivation im Vordergrund, so sind seit etwa zehn Jahren die ökonomisch-pragmatische (Deutsch für den Beruf) und die politische (Deutsch als Verkehrs- und Rechtssprache in regionalen und supranationalen Zusammenschlüssen und Organisationen) weitestgehend an deren Stelle getreten."

Und weiter unten heißt es:

\begin{abstract}
„Dieser Wandel bedeutet, dass das Lehren und Lernen der deutschen Sprache nicht mehr ausschließlich im Rahmen der germanistischen Institute, und bald mehrheitlich nicht mehr nur eingebettet in einen germanistischen Studiengang, stattfinden, sondern dass Kombinationen von Deutsch mit anderen Disziplinen bzw. ein verstärktes Angebot von Deutsch für Hörer aller Fakultäten und/oder Deutsch als Fremdsprache oder deutschsprachige Studiengänge in den Ingenieur- und Wirtschaftswissenschaften z. B., in Übersetzer- und Dolmetscherkursen etc., die Regel sein werden. Organisatorisch hat dies vielfach die Umwandlung einer, Deutschabteilung' in ein ,Service Department zur Folge. Dies widerstrebt naturgemäß den meist in traditionell philologischen Bahnen ausgebildeten und mit einem eher wissenschaftlichen Selbstverständnis arbeitenden ausländischen Germanisten."
\end{abstract}

Ob wir uns damit abfinden und vielleicht die These von Westhoff (2001, S. 7) teilen sollten, Auslandsgermanistik solle sich, wolle sie überhaupt überleben, unbedingt in Richtung Deutschlandstudien hin bewegen, und inwiefern das überhaupt noch mit der Ansicht von Siguan (1998, S. 68) vereinbar ist, wir sollten uns weigern, strikte Berufsausbildung zu betreiben, utilitaristisch zu denken und unsere Existenz als Philologen dauernd rechtfertigen zu müssen, ist eine Frage, auf die es meiner Ansicht nach derzeitig noch keine eindeutige Antwort gibt. Unser Problem ist jedoch gerade auch ein zeitliches: Wir brauchen, um darüber nachzudenken und um die bestmögliche Lösung dafür zu finden, nicht nur Mittel, sondern auch eine Zeit, die man uns vielleicht gar nicht gewähren wird. Und wir laufen somit Gefahr, aus reinem Pessimismus von der in den letzten Jahren erreichten Dynamik abzuweichen. Zum Teil sind ja gerade in den neunziger Jahren, mit dem Aufschwung der Germanistik in Spanien zusammenfallend, auch die Verlage zu neueren Ein-

${ }^{18}$ Mittlerweile auch an den Universitäten Alacant und Cádiz, wo es an sich kein Germanistikstudium als solches gibt. 
sichten gelangt. Und ich meine damit keineswegs spanische Verlage, die für $\mathrm{DaF}$ und Germanistik allgemein immer nur ein sehr schwaches oder überhaupt kein Interesse gezeigt haben, sondern die großen deutschen Lehrbuchverlage. Noch nie hatten sie so viel übersetzt, so viel - wie sie es gern ausdrücken - regionalisiert, natürlich immer nur in Bezug auf das Spanische; das Katalanische, das Galicische und schon gar das Baskische existieren für sie kaum, was aus rein wirtschaftlichen Gründen wohl als verständlich betrachtet werden darf, aber nicht gerade von Sprachsensibilität zeugt. Wo früher in den Veröffentlichungen der Verlage Universalität und Einsprachigkeit Gebot waren, waren plötzlich Einbeziehung der Mutter-, bzw. der Pseudomuttersprache und auch Pseudointerkulturalität angesagt. Es sind in diesen letzten Jahren nicht nur eine ganze Reihe von sogenannten Anfängergrammatiken und sonstigen Grammatiken übersetzt worden - was ein Anfänger mit einer auf Deutsch verfassten Grammatik anfangen konnte, blieb immer rätselhaft $-{ }^{19}$ sondern es ist daneben auch versucht worden, vor allem die die Lehrbücher begleitenden Arbeitsbücher zu regionalisieren, auf eine Art und Weise, die mehr von einem selbstverständlich legitimen Verkaufsdrang als von einem wirklichen Interesse zeugt. ${ }^{20}$ Terminologieabsurditäten und Fokussierung von Allgemeinplätzen wie die Gegenüberstellung der vermeintlichen Pünktlichkeit aller Deutschen und der außer Zweifel stehenden Unpünktlichkeit aller Spanier sind die Folgen gewesen. Hier erweist sich eine Kluft zwischen Germanistik und Verlagen, bei der man nicht genau weiß, bei wem die Schuld liegt, die aber auf jeden Fall durch eine engere und vor allem durch eine seriösere Zusammenarbeit überwunden werden sollte. Vielleicht könnten dadurch sogar bessere und tatsächlich landesspezifische Lehrbücher entstehen, die es in Spanien leider nicht gibt und die z. B. darauf Rücksicht nehmen könnten, dass es bei einem Deutschlerner, der das Spanische als Ausgangssprache hat, wenig Sinn ergibt, das Zustandspassiv als grammatisches Problem erklären zu wollen und dass es auch im Spanischen Funktionsverbgefüge und Abtönungspartikeln gibt.

Ich habe zu Anfang den katalanischen Journalisten Carlos Sentís zitiert und möchte es zum Abschluss noch einmal tun. Vor vier Jahren schrieb er in Bezug auf Katalonien, dass die Generationen des 21. Jahrhunderts über unsere Probleme mit der Zweisprachigkeit - Katalanisch und Spanisch - nur lachen würden, da sie selber schon alle mehrsprachig sein würden (La Vanguardia, 19.11.1997). Aus der Sicht eines Katalanen, der als Sprecher einer Minderheitssprache gegenüber allen Sprachen eine besondere Sensibilität seitens der Politiker erwartet, möchte ich nur hoffen, dass er Recht behält, dass die sogenannte Muttersprache-plus-zwei-Regel, auf die ich mich vorhin schon bezogen habe, nicht nur reines Palaver bleibt, und dass diese Mehr-

19 Z. B. Dreyer, Hilke/Schmitt, Richart (1994 u. 2001) u. Reimann, Monika (1999).

${ }^{20}$ Z. B. Rall, Marlene u. Dietrich (1991), Bock, Heiko et al. (1995), Funk, Hermann et al. (1996), Lüthi (1997) und Schafgans (1998). 
sprachigkeit nicht nur dazu dienen wird, in einer immer stärker globalisierten Welt über Geschäfte zu sprechen. Dazu brauchen wir vielleicht doch nur Englisch.

\section{Literatur}

Acosta, Luís (1997): Germanistik und Deutsch an den spanischen Hochschulen. Institute, Lehrkräfte und Studenten 1997. Madrid.

Ammon, Ulrich (1993): Empirische Untersuchungen. In: Born, Joachim/Stickel, Gerhard (Hg.): Deutsch als Verkehrssprache in Europa. Jahrbuch Institut für Deutsche Sprache 1992. Berlin/New York.

Bock, Heiko et al. (1995): Themen neu. Lehrwerk für Deutsch als Fremdsprache. Libro de ejercicios 1 . Ismaning.

Dreyer, Hilke/Schmitt, Richard (1994): Prácticas de gramática alemana. (Lehr und Übungsbuch der deutschen Grammatik). Ismaning.

Dreyer, Hilke/Schmitt, Richard (2001): Prácticas de gramática alemana - Edición revisada y aumentada. (Lehr und Übungsbuch der deutschen Grammatik - Neubearbeitung). Ismaning.

Funk, Hermann et al. (1996): Sowieso. Curso de Alemán como Lengua Extranjera para Jóvenes. Libro de ejercicios 2. Berlin/München/Wien/Zürich/New York.

Hengst, Hiltrud (2001): Secciones bilingües de alemán en la enseñanza pública. Un proyecto pionero en Andalucía. In: Magazin 10, S. 70-73.

Lüthi, Piet (1997): Stufen International. Curso de Alemán para Jóvenes y Adultos 1. Cuaderno de ejercicios. Barcelona.

Muñoz, Carmen (1999): The effects of age on instructed foreign language acquisition. In González, S. et al. (Hg.): Essays in englisch language teaching. Universidad de Oviedo.

Muñoz, Camen (2001): Factores escolares e individuales en el aprendizaje formal de un idioma extranjero. In: Pastor, S./Salazar, V. (Hg.): Tendencias y líneas de investigación en la adquisición de segundas lenguas. Universidad de Alicante.

Pfeiffer, Michael (1998): Auf dem Wege der Besserung: Deutsch in Spanien. In: DAAD (Hg.): Germanistentreffen Deutschland-Spanien-Portugal, 13-18.09.1998. Dokumentation der Tagungsbeiträge. Bonn. S. 33-43.

Rall, Marlene u. Dietrich (1991): Sprachbrücke. Arbeitsbuch Deutsch-Spanisch. München.

Reimann, Monika (1999): Gramática esencial del alemán. (Grundstufengrammatik für Deutsch als Fremdsprache). Ismaning.

Schafgans, Hans H. (1998): Stufen International. Curso de Alemán para Jóvenes y Adultos 2 . Cuaderno de ejercicios. Barcelona.

Siguan, Marisa (1998): Überlegungen zum Germanistikstudium in Spanien: am Beispiel der Universität Barcelona. In: DAAD (Hg.): Germanistentreffen Deutschland-Spanien-Portugal, 13-18.09.1998. Dokumentation der Tagungsbeiträge. Bonn. S. 61-70.

Stassen, Manfred (2001): Auswertung der „Länderkonzeption deutsche Sprache/Germanistik“ (LKZ). Kurzfassung der Ergebnisse und Bewertungen (online). <http:// www.daad.de/germanistik/auswertung_laenderkonzeption.pdf.> Eingesehen am 5.3.2002.

Westhoff, Gerard (2001): IDV 1993-2001 oder: DaF geht in den Spagat. In: IDV-Rundbrief. Heft 66, April. S. 4-8.

Zurdo, Maria Teresa (2001): Kontrastive Analysen Deutsch-Spanisch: eine Übersicht. In: Helbig, Gerhard et al. (Hg.): Deutsch als Fremdsprache: ein internationales Handbuch. Berlin/New York. S. 375-377. 